Family and Neighbourhood Risk and Children's Problem Behaviour: The

Moderating Role of Intelligence

Eirini Flouri*, Emily Midouhas*, \& Heather Joshi**

*Department of Psychology and Human Development, UCL Institute of Education,

University College London

**Department of Quantitative Social Science, UCL Institute of Education, University College London

Correspondence: Eirini Flouri, Department of Psychology and Human Development, UCL Institute of Education, University College London, 25 Woburn Square, London WC1H 0AA, UK. Email: e.flouri@ioe.ac.uk 


\title{
Family and Neighbourhood Risk and Children's Problem Behaviour: The Moderating Role of Intelligence
}

\begin{abstract}
General cognitive ability has been shown to buffer the effects of family adversity and poverty on emotional and behavioural problems in school age children. Yet, little is known about whether 'intelligence' can protect younger children or change the problem trajectories of at-risk children. We modelled simultaneously the effects of family poverty, neighbourhood poverty and adverse family events on children's trajectories of emotional and behavioural problems at ages 3,5 and 7. We then tested the role of general intelligence both in changing the trajectories of problems and in buffering the effects of these risk factors at each age, and explored gender differences in the expected protective effect. We analyzed 16,916 children from the UK Millennium Cohort Study. General intelligence was derived from principal components analysis of several cognitive ability measures at ages 3,5 and 7 . Although general intelligence was not associated with the growth of at-risk children's problems over time, it was associated with the level of positive emotional and behavioural outcomes and conferred concurrent protection from risk. At age 5, poor children with higher general intelligence had fewer emotional problems than similarly poor children with lower intelligence. Children exposed to family adversity were less likely to have emotional problems at any age if they had higher general intelligence. Higher general intelligence was also related to fewer behavioural problems for children experiencing family adversity at age 5 , but not at ages 3 or 7 . General intelligence moderated the effect of neighbourhood poverty on behavioural problems at ages 3 and 7 , and its effect on emotional problems at age 5 . In general, the protective effects of general intelligence, especially for emotional outcomes,
\end{abstract}


applied mainly to girls. General intelligence appears to assist children in building resilience to both family and neighbourhood risk across childhood.

Keywords: cumulative risk; emotional and behavioural problems; intelligence; Millennium Cohort Study; resilience 


\section{Family and Neighbourhood Risk and Children's Problem Behaviour: The Moderating Role of Intelligence}

\section{Introduction}

Poverty and family adversity can both predict and exacerbate children's emotional (internalising) and behavioural (externalising) problems (Bradley \& Corwyn, 2002; Drukker, Kaplan, Feron, \& van Os, 2003; Goodnight et al., 2012; Kohen, Leventhal, Dahinten, \& McIntosh, 2008; Reiss, 2013). However, children exposed to these risk factors also vary in their outcomes (Kim-Cohen, Moffitt, Caspi, \& Taylor, 2004), and therefore some children escape their effects. This 'resilience' may be due to certain individual characteristics, family qualities or environmental influences, likely working together to forge protection through a dynamic process (Rutter, 2013).

An individual-level protective factor for such emotional and behavioural resilience in school age children is general cognitive ability or intelligence ('g'; Breslau, Lucia, \& Alvarado, 2006; Masten et al., 1999; Riglin et al., 2015; Fergusson \& Lynskey, 1996). For example, Riglin et al. (2015) found that higher ability buffered the negative impact of adverse life events on depressive symptoms among adolescents in a community sample and a sample at-risk for depression. In both variable-centred and person-centred analyses, Masten et al. (1999) demonstrated that general intelligence was associated with lower risk of antisocial behaviour among adolescents experiencing adversity, even chronic adversity, and that 'resilient' individuals had levels of intelligence (average to high) similar to their counterparts who experienced less adversity.

Children facing potentially stressful experiences, such as adverse family circumstances or the challenges associated with living in poverty in the home or the neighbourhood, may be 
particularly benefited by having higher general intelligence. At-risk children with higher general intelligence may have greater problem-solving aptitude, enabling them to assess threats effectively and use available information to find solutions for stressful situations (Masten et al., 1999). They may also be more likely to find meaning in their adversity and positively reframe their situation (Riglin et al., 2015). Additionally, children with higher intelligence may have a greater capacity for seeking out healthier or more advantageous environments (Gottfredson \& Deary, 2004). Children with lower general intelligence, on the other hand, may be less equipped in these ways to cope with stressful situations (Barnett, Salmond, Jones, \& Sahakian, 2006; Koenen et al., 2009). Yet, it is not clear from these studies if intelligence can change the development of problem behaviour of at-risk children, or whether any protective effect depends on developmental period. It is also unclear if such 'protection', usually seen in older children and adolescents, can be evidenced in early childhood when the capacity to self-select into more advantageous circumstances and contexts is more limited. In this study, we attempted to address these issues. We investigated if intelligence is a protective factor for young children exposed to poverty and adversity, and, if it is, whether it changes the problem behaviour trajectories of such at-risk children or simply differentiates them at given ages. We also tested whether the expected moderator effects of general intelligence differ by the child's gender. There is some evidence that, at least in adolescence, cognitive ability is both more promotive (Weeks et al., 2014) and more protective for girls' compared to boys' mental health (Riglin et al., 2015), although the reasons for this gender difference are unclear.

\section{The Present Study}

We used data from the UK's Millennium Cohort Study, a large cohort of families with young children, followed longitudinally from age 9 months. We explored the longitudinal associations between risk and children's general intelligence and emotional and behavioural 
adjustment at all early and mid-childhood data sweeps with data on these measures, corresponding to ages 3,5 and 7. The main risk factors, all time-varying at these ages, were number of potentially adverse life events the family experienced between sweeps, neighbourhood poverty and family poverty.

We adjusted for selected family/parent and child characteristics in order to rule out confounders of the relationship between poverty and adversity and child outcomes. The family-level covariates were mother's education and parental involvement in learning. Mother's education is strongly related to both family risk and child behaviour (Evans \& English, 2002). At-risk children are also more likely to have parents who are less involved in their learning (Hornby \& Lafaele, 2011), and low parental involvement is related to child problem behaviour (Wang \& Sheikh-Khalil, 2014). Our child-level covariates were gender, ethnicity and self-regulation. Girls, in general, are at lower risk of behavioural problems than boys (Egger \& Angold, 2006). The main ethnic minority groups in the UK have similar or lower rates of emotional, behavioural and hyperactivity problems than white British children (Goodman, Patel, \& Leon, 2008), despite experiencing more poverty (Platt, 2007). Selfregulation is related negatively to emotional and behavioural problems in children (Eisenberg, Spinrad, et al., 2010; Lengua, 2003) and positively to their emotional and behavioural resilience to several risk factors, including poverty (Buckner, Mezzacappa, \& Beardslee, 2003; Flouri, Midouhas, \& Joshi, 2014). By including self-regulation, the capacity to direct and focus one's actions and/or attentions to meet one's goals, we were also able to isolate the effect of general intelligence from executive functions and self-regulatory capacities which are positively associated with cognitive ability (Bornstein, Hahn, \& Suwalsky, 2013; McClelland et al., 2007; Nisbett et al., 2012).

Moreover, as we estimated the effect of risk at the neighbourhood level, it was necessary to account for selection bias. Selection bias occurs when the mechanism sorting 
families into neighbourhoods is not independent from the outcome studied (Ginther et al., 2000). For example, in the case of neighbourhood income, families who are more educated are more likely to live in high-income areas, and families who are less educated are more likely to live in low-income areas. In addition to mother's education, family poverty and adverse life events (which included family disruption, worsening financial circumstances and maternal depression) were associated with neighbourhood sorting. Accounting for neighbourhood selection allows the estimates for neighbourhood to reflect the influence of locality over and above the circumstances of the individual family.

\section{Methods}

\section{Participants and Procedure}

The Millennium Cohort Study (MCS; www.cls.ioe.ac.uk/mcs) is a longitudinal survey drawing its sample from all births in the UK over a year, beginning on 1 September 2000 (Plewis, 2007). The MCS sample design over-represented families living in areas of high child poverty, areas with high proportions of ethnic minority populations across England, and the three smaller UK countries. Ethical approval for the MCS was gained from NHS MultiCentre Ethics Committees, and parents gave informed consent before interviews took place. Sweeps 1-4 took place when the children were around 9 months, and 3, 5 and 7 years, respectively. Emotional and behavioural problems were measured at Sweeps 2-4. Our sample was families whose children had data on internalising problems or externalising problems in at least one of Sweeps 2-4 $(n=16,916)$, the majority of the MCS families $(n=19,244)$.

\section{Measures}

Internalising and externalising problems were measured at ages 3, 5 and 7 with the main parent-reported Strengths and Difficulties Questionnaire (SDQ; Goodman, 1997). The SDQ is a 25-item scale measuring four domains of difficulties (hyperactivity, emotional 
symptoms, conduct problems and peer problems) and prosocial behaviour. Item responses range from 0 to 2. In line with recommended practice (Goodman, Lamping, \& Ploubidis, 2010), the internalising problems scale comprised the 10 items from the emotional symptoms and peer problems subscales, and the externalising problems scale was derived from the 10 items from the hyperactivity and conduct problems subscales. Scores for each 10-item scale may range from 0 to 20 . In our sample, internal consistency was at acceptable levels, and in line with other SDQ research (Stone, Otten, Engels, Vermulst, \& Janssens, 2010). Cronbach's alpha values across the three sweeps ranged from .61 (at age 3) to .72 (at age 7) for internalising, and from .78 (at age 3 ) to .80 (at age 7) for externalising problems.

Family poverty, operationalized as socio-economic disadvantage (SED), was measured (as in Malmberg \& Flouri, 2011) as the sum of four binary indicators of the family's economic deprivation. Given incompleteness of measured family income, this SED score captures poverty and its associated material conditions more generally, providing a broader view of family-level socio-economic risk factors. The four items were overcrowding $(>1.5$ people per room excluding bathroom and kitchen), not owning the home, receipt of meanstested income support, and income poverty (below a line set as equivalised net family income at $60 \%$ of the national median). We created a time-varying summary score of the four SED items (or of all valid items where any were missing) ranging 0-4.

Adverse life events (ALE) at ages 3, 5 and 7 were measured as the number (out of eleven) of potentially stressful life events experienced by the family between two consecutive sweeps. The events, derived from available MCS data and based on Tiet et al.'s (1998) Adverse Life Events Scale, were: family member died, negative change in financial situation, new stepparent, sibling left home, child got seriously sick or injured, divorce or separation, family moved, parent lost job, new natural sibling, new stepsibling, and maternal depression 
(treated for or diagnosed with depression). At each sweep, the number of events occurring since the previous sweep was summed to form a total ALE score.

Neighbourhood median income (NMI) at ages 3, 5 and 7 was measured for each Lower layer Super Output Area (LSOA) ${ }^{1}$ with data from Experian, drawn from multiple sources, including the Census and market research and public sector datasets (Experian, 2011). For Sweep 2, the 2004 estimate of NMI was taken; for Sweep 3, that for 2005 (2006 not being available); and for Sweep 4, that for 2008. Due to the positively skewed distribution of NMI, we used its logarithm in all statistical models.

To measure general intelligence $(I Q)$ at ages 3, 5 and 7, regression factor scores were derived at each age from principal components analysis of multiple age-adjusted ability assessment scores. Then each factor score was transformed into a standardized IQ score with a mean of 100 and a standard deviation of 15 (Hanscombe et al., 2012). These multiple wellvalidated assessments are thought to measure the underlying general intelligence factor (or ' $\mathrm{g}$ '), which, at least in adults, has been shown not to be dependent on the use of specific mental ability tasks (Johnson, Bouchard, Krueger, McGue, \& Gottesman, 2004). Therefore, the comparability of IQ scores over time is here theoretically inferred, in line with previous research on the trajectories of ' $g$ ' in children (von Stumm \& Plomin, 2015). At Sweep 2, the two MCS cognitive assessments comprised the Bracken School Readiness AssessmentRevised, which assesses children's 'readiness' for formal education by testing their knowledge and understanding of basic concepts (Bracken, 1998), and the second edition of the British Ability Scales (BASII; Elliot, Smith, \& McCulloch, 1996) Naming Vocabulary scale, which measures expressive language. At Sweep 3, ability was assessed, on three scales with the BAS Naming Vocabulary, BAS Pattern Construction (measuring spatial problem

\footnotetext{
${ }^{1}$ LSOAs cover around 1,500 inhabitants, with boundaries drawn to maximise social homogeneity.
} 
solving) and BAS Picture Similarities (measuring non-verbal reasoning) scales. At age 7 , MCS measured cognitive skills by three scales: with the BAS Pattern Construction, again, maths achievement - assessed with the National Foundation for Educational Research Progress in Maths - and BAS Word Reading (measuring educational knowledge of reading) scales.

Key covariates were both family- and child-level, as explained. The family-level variables were maternal education [University degree (by child's age 7) or not] and parental involvement [measured at ages 3,5 and 7 by the frequency with which the main parent reads to the child, ranging 1 (every day) to 5 (not at all)]. The child-level variables were gender, ethnicity and self-regulation. Self-regulation was measured (at ages 3, 5 and 7) with a scale from the Child Social Behaviour Questionnaire (CSBQ), based on the Adaptive Social Behavior Inventory (Hogan, Scott, \& Bauer, 1992). The CSBQ was developed and constructvalidated as part of the Effective Provision of Pre-School Education project for England (Sammons et al., 2004) and Northern Ireland (Melhuish et al., 2004). It has good internal consistency, established with samples of children aged 5. In the multi-purpose MCS, the number of CSBQ items on self-regulation was restricted to five, completed by the main parent. Items, on 3-point scales, range from 1 to 3 . The items ('likes to work things out for himself/herself', 'does not need much help with tasks', 'chooses activities on his/her own', 'persists in the face of difficult tasks', and 'moves to new activity after finishing task') measure children's capacity for independently guiding and controlling their actions and behaviour. At each sweep, we calculated an average time-varying score (ranging 1-3) of items with valid data in the scale. Cronbach's alpha values ranged, across sweeps, from .57 (at age 3) to .66 (at age 7).

\section{Analytic Strategy}


First, we investigated whether the families in our analytic sample $(n=16,916)$ were different $($ at $\mathrm{p}<.05)$ from those not in it $(\mathrm{n}=2,328)$ on our study variables. Next, we explored the shape of the children's average trajectories of externalising and internalising problems, which, as discussed below, was curvilinear. Following this, we inspected the correlations between our main (risk, moderator and outcome) variables. We then explored levels and patterns of missingness in our covariates to decide on our approach to dealing with missing data. Finally, we fitted three-level growth curve models which enabled us to avoid the underestimation of standard errors due to the hierarchical nature of our data in having repeated measures (at ages 3, 5 and 7) of externalising and internalising problems (Level 1) nested in children (Level 2) nested in areas (Level 3). We accounted for area clustering at the level of ward on which the MCS survey design was built. In all our conditional models (i.e., Models 2-7), we adjusted for stratum (a Level 3 variable) to reflect the stratified sample design. Growth curve models allowed us to estimate the average level of problems at a particular time-point and the average growth rate in problems over time. By specifying a random slope on the child's age to allow for changes in problems across time varying between children, we could also model individual trajectories of problems from ages 3 to 7 . We fitted both fixed and random linear slopes, and included a fixed quadratic term to account for the curved shape of children's average trajectories.

The full sequence of models estimated is outlined in Table 1. Model 1 (the unconditional model) investigated the average levels and growth of externalising and internalising problems by regressing them on age in years and its square. We carried out models initially with age grand mean centred at 5.22 years. Grand mean centring age at the 'midpoint' minimises the correlation between age and age-squared, thus stabilising the estimation. Model 2 added the three risk factors, specified to be related to the intercept and slopes (linear and quadratic) of externalising and internalising problems. This enabled us to 
examine whether levels of problems at around age 5 and rate of change in problems over time shifted with SED, ALE and NMI. Model 3 added the child and family covariates. Model 4 introduced the proposed moderator, IQ, allowing it to predict the intercept and slopes of externalising and internalising problems. Models 5, 6 and 7 investigated the interaction between each risk factor and IQ, also specified to predict the intercept and slopes. To test for differences at baseline and at the latest sweep, we also ran separate models centring child's age at the average age at Sweep 2 (3.14 years) and then at the average age at Sweep 4 (7.23 years). This allowed us to evaluate the IQ effects at the starting and end surveys in this series, as well as at the grand mean. Lastly, we ran the full set of models for boys and girls separately, centring age at $3.14,5.22$ and 7.23 years.

(Table 1)

\section{Missing Data Analysis and Imputation}

There was some missingness on our study variables in the analytic sample (children who had emotional and behavioural problem data in at least one of Sweeps 2-4). Of our sample cases, $30-36 \%$ had some missing data on covariates across sweeps, and 6-8\% of values of covariates were missing across sweeps. To deal with missingness, we multiply imputed missing data for the covariates (but not the dependent variables). Given that a range of $6-8 \%$ of values were missing across sweeps, we generated 5 imputed datasets (Allison, 2009; Graham, Olchowski, \& Gilreath, 2007) in SPSS22 using the Markov Chain Monte Carlo procedure. In the imputation model we included all covariates as predictor and predicted variables. We fitted our models in Stata14 using the MI estimate command, which performs individual analyses for each of the imputed datasets, collects estimates of coefficients and their variance-covariance estimates, applies Rubin's combination rules (Rubin, 1996) to the collected estimates, and reports pooled results.

\section{Results}




\section{Bias Analysis and Descriptives}

The children in the analytic sample differed from those in the non-analytic sample on some study variables (Table 2), suggesting that our analytic sample was, as expected, somewhat more advantaged. More specifically, the children in the analytic sample were significantly more likely to be white, experience greater parental involvement and have higher IQ and higher self-regulation. They also experienced fewer stressful life events, but at age 3 only, as well as lower family and neighbourhood poverty at all three ages. In the analytic sample, all three risk factors (SED, ALE and NMI) were significantly related to both IQ and emotional and behavioural problems (Table 3), although the strength of the association differed by type of risk factor. Poverty (at both family and neighbourhood) was more strongly related to all three child outcomes than ALE. ALE also had a weak association with both SED and NMI.

(Tables 2 and 3)

\section{Model Results}

We began by running models with age grand mean centred at 5.22 years. The unconditional model (Model 1, Table 4) revealed that, in the analytic sample, at an annual rate around age 5, children's internalising and externalising difficulties decreased by -0.04 and -0.48 points on the SDQ scale per annum, respectively. The quadratic term for age showed that the downward slopes were steeper before age 5, particularly for externalising problems. From age 3, internalising problems fell by about one third of a point on the SDQ scale, and externalising by about 1.3. The fitted trajectories for both types of stopped falling after age 5 (at 5.5 and 6.0 years, respectively), turning slightly upward before age 7 . The within-child, between-child and between-ward variance was larger in externalising than in 
internalising problems, suggesting that, externalising problems varied more over time, and differed more between both families and areas.

(Table 4)

In Model 2, all three risk factors were associated, in the expected direction, with internalising and externalising problems at around age 5, with SED having the largest effect of the three (externalising: $\mathrm{t}=18.89$; internalising: $\mathrm{t}=15.58$ ). Furthermore, SED was associated with a linear change in externalising problems with each year, and number of ALE was related to a non-linear annual change in externalising problems. NMI was associated with both linear and non-linear change in externalising problems over time. As for internalising problems, SED was unrelated to the rate of change in problems. However, ALE and NMI were associated with annual change in internalising problems.

Adding child and family covariates to the models (Model 3) did not attenuate either the main or the interactive (with age) effects of the three risk factors on externalising or internalising problems. The child and family covariates were, in general, associated with both types of problem behaviour. Specifically, girls, black children, children with greater selfregulation, those with university-educated mothers and those with parents who read to them more frequently had fewer externalising problems. There were fewer internalising problems for children from mixed, Pakistani/Bangladeshi or 'other' ethnic backgrounds; with greater self-regulation, University-educated mothers and parents who read to them more frequently. In Model 4 (Table 4), the main effect of IQ was significantly associated with both types of problems (internalising: $\mathrm{t}=-8.24$; externalising: $\mathrm{t}=-13.20$ ). IQ was also related to the nonlinear rate of change in both sets of problems over time (internalising: $\mathrm{t}=-3.02$; externalising: $t=-3.69)$, but not to the linear rate of change in either . The inclusion of IQ did not attenuate either the main or the interactive effects of our risk factors. IQ, therefore, did 
not explain away the association of adverse life events, poverty or neighbourhood deprivation with externalising or internalising problems. Nor did it explain why these risk factors were related to the changes over time in internalising and externalising problems.

Models 5-7 tested the expected moderator effects of IQ. The effects of SED, ALE and NMI on internalising problems at central age were weaker in children with greater IQ. In addition, the effect of the number of ALE on externalising problems at central age was moderated by IQ. However, we did not find any moderator effects on the annual change of either internalising or externalising problems over time. We then ran Models 1-7 with the age variable centred at 3.14 and 7.23 years, to test whether the effects of IQ depended on the developmental period of the child. At around age 3, IQ moderated the effect of NMI on externalising problems and that of number of ALE on internalising problems. At around age 7, the effect of NMI on externalising problems was weakened for those with higher IQ, as was the effect of number of ALE on internalising problems. IQ did not alter the effect of SED on child outcomes at either age 3 or age 7 .

To unpack the interactions between our risk factors and IQ, we plotted the predicted values for internalising and externalising score trajectories, based on Models 5, 6 and 7, for illustrative cases with high, average and low IQ by experience of risk (Figures 1-4). High IQ was defined by a score of two standard deviations above the mean of 100 (i.e., 130). Average IQ corresponded to a score of 100. Low IQ referred to a score of two standard deviations below the mean [i.e., 70, the cutoff for intellectual disability according to the American Psychiatric Association (2013)]. High and low levels of risk factors corresponded to the $90^{\text {th }}$ and $10^{\text {th }}$ percentiles, respectively.

Turning first to internalising problems, Figure 1 displays the significant interaction between SED and IQ at age 5. As can be seen, a poor (high SED) child with a high IQ has 
fewer internalising problems around age 5 (predicted score of about 2.5) than a poor child with a low IQ (predicted score of about 3.5). Hence, these two children have a gap of roughly 1 point on the SDQ scale. A poor child with an average IQ has a predicted score of around 3, that is, a score that lies midway between the latter two children. A non-poor (low SED) child with a low IQ has a predicted score around 2.5, nearly indistinguishable from the poor/high IQ child at age 5. Moreover, the non-poor child with an average IQ and the non-poor child with a high IQ have the fewest internalising problems with about a .2 point gap between them on the scale.

Figure 2 shows the differences in predicted internalising problem scores by number of ALE (high and low) and level of IQ (high, average, low) at ages 3, 5 and 7. At age 5, there are roughly .5 point gaps in the internalising scale between the high-adversity children with low, average and high IQ. These gaps are slightly larger at ages 3 and 7. For the lowadversity children (with high, average and low IQ) at age 3 there are differences of roughly .2-.3 points on the scale. These gaps narrow around age 5, only to widen again from around age 6. Across time, the trajectories for the low-adversity children stay at or below the trajectory for the high-adversity/average IQ child, with the two high IQ children having similar scores until age 6 when they begin to diverge in favour of the low-adversity/high IQ child.

Turning to externalising problems (Figure 3), the high-adversity/low IQ child appears to have, as expected, the highest predicted scores. This was 6.2 at age 5 , where we found a significant interaction of ALE and IQ. The high-adversity/average IQ child has roughly .7 fewer points than the high-adversity/low IQ child, and the high-adversity/high IQ child has around .7 fewer points than the high-adversity/average IQ child. As with internalising problems, the two high IQ children have the lowest internalising scores (around 5). 
Lastly, Figure 4 depicts the age 3 and age 7 interactive effects of NMI and IQ on externalising problems. At age 3, the low NMI/low IQ child stands out from all other children with an externalising score around 8.7. This externalising score is close to the borderline abnormality cutoff of 9 (Goodman, 1997), indicating that a low IQ child living in a poor neighbourhood is at risk of having clinical levels of externalising problems. However, the children with either an average or a high IQ who live in a low-income neighbourhood have scores below this, around 7.5 (average IQ) and 6.9 (high IQ), respectively. The children in non-poor neighbourhoods have scores ranging 6.5 to 7.6, overlapping with those of the average or high IQ children in poor neighbourhoods. At age 7, the differences in the externalising scores of children in poor neighbourhoods with high, average or low IQ are of similar magnitude to those at age 3, although externalising problems in all children, irrespective of their levels of IQ or neighbourhood poverty, have improved since age 3.

(Figures 1-4)

\section{Gender Differences}

To test for gender differences in the moderator effects of intelligence, we ran Models 17, centring age at $3.14,5.22$ and 7.23 years - the mean ages of the age 3,5 and 7 surveys separately for boys and girls. At around age 3, IQ had no significant moderator effects for boys. However, for girls, it weakened the relationships between family risk (SED and ALE) and their externalising problems, as well as those between internalising problems and both NMI and number of ALE. At around age 5, at the beginning of primary school, IQ moderated the impact of NMI on boys' internalising problems. For girls, it moderated the effects of all three risk factors on internalising problems. However, it did not moderate the effects of any risk factor on either boys' or girls' externalising problems. At around age 7, there were no significant moderator effects for girls. For boys, IQ moderated the impact of number of ALE on internalising problems. 
We then explored why, at age 7, IQ moderated the association between adverse life events and internalising problems in boys but not girls, whereas IQ more consistently moderated risk factors for girls at ages 3 and 5. The rising importance of family structural changes (such as a new sibling, divorce or separation, and a new stepparent) from ages 3 to 7 may be one reason for boys' sensitivity at age 7 . These are captured in the adverse life events variable. Another reason may be the gender difference in the strength of the relationship between the number of adverse life events and internalising problems. Looking at the main effects of adverse life events on internalising problems in adjusted models, and at basic correlations between adverse life events and internalising problems, at age 7, the relationship between adverse life events and internalising problems was stronger for boys than for girls, suggesting that life adversity had a greater impact on boys' emotional problems than on girls' at this age. At ages 3 and 5, we did not find this disparity. There is some research that provides modest support for the idea that boys are more negatively affected by divorce (Amato \& Keith, 1990; 2001), which is more common among the included adverse life events after age 5. In our sample, we found, using correlations, that the relationship between experiencing divorce or separation between ages 5 and 7 and internalising problems was statistically significant for boys but not for girls. Other research has also shown that some well-known indices of cumulative risk or (family-level) environmental adversity, such as Rutter's (1978; including inter-parental conflict, low social class, large family size, paternal criminality, maternal mental disorder, and experience of being in 'care') have stronger effects on boys' compared to girls' emotional and behavioural problems (Biederman, Faraone, \& Monuteaux, 2014). If boys are more vulnerable to the effects of adverse life events than girls in mid-childhood, then a protective factor like IQ is likely to play a more important role in their coping with adversity. 


\section{Discussion}

The consequences of life stress and poverty for child development are not inevitable, and some children appear to beat the odds stacked against them. In some cases, the agency of the child, parents or other adults may help explain why some children do better than expected given their level of exposure to such risk factors. In this paper, we explored the role of the child's intelligence in promoting psychological adjustment and resilience to the risk factors of adverse life events, family poverty and neighbourhood poverty. The role of IQ in resilience to such risk has been explored in research with school age children and adolescents, but not with young children. Our study, in a large UK sample, tested the role of IQ in moderating the effects of these risk factors on children's emotional (internalising) and behavioural (externalising) problems from early-to-middle childhood. We found that, even after controlling for self-regulation and parental socio-economic background, IQ - measured as general intelligence (' $\mathrm{g}$ ') - was moderately predictive of concurrent emotional and behavioural adjustment. Importantly, it acted as a buffer of the negative impact of family poverty, adverse life events and neighbourhood poverty, suggesting that family and neighbourhood risk may have weaker influences on a child's problem behaviour if the child can use her problem-solving aptitude to manage the related stress (Masten et al., 1999).

Perhaps our most noteworthy finding is that IQ, consistently across early-to-middle childhood, moderated the effect of adverse life events on concurrent internalising symptoms. Therefore, in both early and middle childhood, IQ seems to be important in protecting children exposed to family stressors from internalising symptoms. Nevertheless, some of the moderator effects of IQ only applied to age 5. Specifically, IQ buffered the negative impact of adverse life events on concurrent externalising problems only at age 5. It weakened the effect of both neighbourhood and family poverty on internalising problems, again at age 5 
only. At around age 5, children in the UK start primary school and must learn to interact with peers and cope with a structured extra-familial setting focussing on learning rather than play. This transition period may be a challenge for children, particularly those who are experiencing additional life stressors such as poverty, divorce or parental depression. It may be at this juncture that IQ plays an important role in helping children to cope with these challenges.

Nonetheless, IQ did not alter the trajectories of at-risk children's problems. More and less intelligent children exposed to our family and neighbourhood risk factors seemed to take parallel paths in their problems across childhood, although the more intelligent children had fewer problems at any given point in the trajectory. This may suggest that whatever benefits IQ has for managing behaviours and emotions are already in effect by the time children are 3 years old, and that IQ continues to help children maintain their relative place on the problem behaviour spectrum as they get older despite continual exposures to risk. We do acknowledge, however, that basing our IQ measure at age 3 on factor scores derived from only two cognitive measures, one of which was a school readiness assessment (Bracken School Readiness Assessment) rather than a mental ability test, may limit the validity of this finding.

As for gender differences, it appears that, in general, the protective effects of IQ, and particularly those on internalising problems in early and middle childhood, were driven mainly by girls. This suggests that building girls' cognitive skills early can help them move on a low problems trajectory. The finding that cognitive ability appeared to have stronger protective effects for girls' compared with boys' mental health is consistent with recent findings with adolescents (Riglin et al., 2015), although the reasons for this gender difference remain unclear. However, for both boys and girls - and in both our earliest and latest 
assessments - IQ had a protective effect from externalising problems, too. IQ appeared to help children maintain behavioural adjustment in the face of neighbourhood poverty in both early and middle childhood.

These findings should be viewed in light of three important study limitations. First, our measure of IQ was derived from standardized assessments of ability that were available at MCS. We only had two measures of ability in Sweep 2, limiting the accuracy of measurement of IQ during this sweep. Second, we were unable to test for measurement invariance for IQ in our samples of boys and girls to confirm whether our gender differences in the protective effect of IQ are genuine. Third, the study is correlational and relied entirely on parental reports of children's emotional and behavioural problems. Despite these limitations, our study showed that even controlling for self-regulation, which is related to executive functioning - in turn likely explaining at least part of the promotive effect of cognitive ability for child adjustment (Diamond, 2013), general intelligence was associated with positive emotional and behavioural outcomes in children experiencing family poverty, neighbourhood disadvantage or family adversity. These effects, already visible at age 3 , the beginning of our study period, persisted into middle childhood. Future research should investigate the mechanisms through which general cognitive ability promotes emotional and behavioural adjustment in at-risk children in early and middle childhood. 


\section{References}

Allison, P. D. (2009). Fixed effects regression models. Thousand Oaks, CA: Sage Publications.

Amato, P. R., \& Keith, B. (1991). Parental divorce and the well-being of children: A metaanalysis. Psychological Bulletin, 110, 26-46.

Amato, P. R., \& Keith, B. (1991). Parental divorce and adult well-being: A meta-analysis. Journal of Marriage and the Family, 53, 43-58.

American Psychiatric Association. (2013). Diagnostic and statistical manual of mental disorders (5th ed.). Washington, DC.

Biederman, J., Faraone, S. V., \& Monuteaux, M. C. (2014). Differential effect of environmental adversity by gender: Rutter's index of adversity in a group of boys and girls with and without ADHD. American Journal of Psychiatry, 159, 1556-1562.

Bornstein, M. H., Hahn, C. S., \& Suwalsky, J. T. (2013). Language and internalizing and externalizing behavioral adjustment: Developmental pathways from childhood to adolescence. Development and Psychopathology, 25, 857-878.

Bracken, B. (1998). Bracken Basic Concept Scale Revised: Examiner's manual. London: The Psychological Corporation.

Bradley, R. H., \& Corwyn, R. F. (2002). Socioeconomic status and child development. Annual Review of Psychology, 53, 371-399.

Breslau, N., Lucia, V. C., \& Alvarado, G. F. (2006). Intelligence and other predisposing factors in exposure to trauma and posttraumatic stress disorder: A follow-up study at age 17 years. Archives of General Psychiatry, 63, 1238-1245.

Buckner, J. C., Mezzacappa, E., \& Beardslee, W. R. (2003). Characteristics of resilient youths living in poverty: The role of self-regulatory processes. Development and Psychopathology, 15, 139-162. 
Diamond, A. (2013). Executive functions. Annual Review of Psychology, 64, 135-168.

Doan, S. N., Fuller-Rowell, T. E., \& Evans, G. W. (2012). Cumulative risk and adolescent's internalizing and externalizing problems: The mediating roles of maternal responsiveness and self-regulation. Developmental Psychology, 48, 1529.

Drukker, M., Kaplan, C., Feron, F., \& Van Os, J. (2003). Children's health-related quality of life, neighbourhood socio-economic deprivation and social capital: A contextual analysis. Social Science and Medicine, 57, 825-841.

Egger, H. L., \& Angold, A. (2006) Common emotional and behavioural disorders in preschool children: Presentation, nosology and epidemiology. Journal of Child Psychology and Psychiatry, 47, 313-317.

Elliott, C. D., Smith, P., \& McCulloch, K. (1996). British Ability Scales Second Edition (BAS II). Administration and scoring manual. London: Nelson.

Evans, G. W. (2003). A multimethodological analysis of cumulative risk and allostatic load among rural children. Developmental Psychology, 39, 924.

Experian. (2011). Household Income 2011: Data profile. Experian Limited, Nottingham, UK. Eysenck, M. W., \& Keane, M. T. (2005). Cognitive psychology: A student's handbook. New York: Psychology Press.

Fergusson, D. M., \& Lynskey, M. T. (1996). Adolescent resiliency to family adversity. Journal of Child Psychology and Psychiatry, 37, 281-292.

Flouri, E., Midouhas, E., \& Joshi, H. (2014). Family poverty and trajectories of children's emotional and behavioural problems: The moderating roles of self-regulation and verbal cognitive ability. Journal of Abnormal Child Psychology, 42, 1043-1056.

Goodman, A., Lamping, D. L., \& Ploubidis, G. B. (2010). When to use broader internalising and externalising subscales instead of the hypothesised five subscales on the Strengths 
and Difficulties Questionnaire (SDQ): Data from British parents, teachers and children. Journal of Abnormal Child Psychology, 38, 1179-1191.

Goodman, A., Patel, V., \& Leon, D. A. (2008). Child mental health differences amongst ethnic groups in Britain: A systematic review. BMC Public Health, 8, 258.

Goodman, R. (1997). The Strengths and Difficulties Questionnaire: A research note. Journal of Child Psychology and Psychiatry, 38, 581-586.

Goodnight, J. A., Lahey, B. B., Van Hulle, C. A., Rodgers, J. L., Rathouz, P. J., Waldman, I. D., \& D'Onofrio, B. M. (2012). A quasi-experimental analysis of the influence of neighborhood disadvantage on child and adolescent conduct problems. Journal of Abnormal Psychology, 121, 95-108.

Gottfredson, L. S., \& Deary, I. J. (2004). Intelligence predicts health and longevity, but why?. Current Directions in Psychological Science, 13, 1-4.

Graham, J. W., Olchowski, A. E., \& Gilreath, T. D. (2007). How many imputations are really needed? Some practical clarifications of multiple imputation theory. Prevention Science, 8, 206-213.

Hanscombe, K. B., Trzaskowski, M., Haworth, C. M., Davis, O. S., Dale, P. S., \& Plomin, R. (2012). Socioeconomic status (SES) and children's intelligence (IQ): In a UKrepresentative sample SES moderates the environmental, not genetic, effect on IQ. PLoS One, 7, e30320.

Hogan, A. E., Scott, K. G., \& Bauer, C. R. (1992). The Adaptive Social Behavior Inventory (ASBI): A new assessment of social competence in high-risk three-year-olds. Journal of Psychoeducational Assessment, 10, 230-239.

Hornby, G., \& Lafaele, R. (2011). Barriers to parental involvement in education: An explanatory model. Educational Review, 63, 37-52. 
Johnson, W., Bouchard, T. J., Krueger, R. F., McGue, M., \& Gottesman, I. I. (2004). Just one $\mathrm{g}$ : Consistent results from three test batteries. Intelligence, 32, 95-107.

Kane, M. J., Hambrick, D. Z., Tuholski, S. W., Wilhelm, O., Payne, T. W., \& Engle, R. W. (2004). The generality of working memory capacity: A latent-variable approach to verbal and visuospatial memory span and reasoning. Journal of Experimental Psychology: General, 133, 189-217

Kim-Cohen, J., Moffitt, T. E., Caspi, A., \& Taylor, A. (2004). Genetic and environmental processes in young children's resilience and vulnerability to socioeconomic deprivation. Child Development, 75, 651-668.

Kohen, D. E., Leventhal, T., Dahinten, V. S., \& McIntosh, C. N. (2008). Neighborhood disadvantage: Pathways of effects for young children. Child Development, 79, 156-169.

Malmberg, L. E., \& Flouri, E. (2011). The comparison and interdependence of maternal and paternal influences on young children's behavior and resilience. Journal of Clinical Child and Adolescent Psychology, 40, 434-444.

Martinez-Torteya, C., Anne Bogat, G., Von Eye, A., \& Levendosky, A. A. (2009). Resilience among children exposed to domestic violence: The role of risk and protective factors. Child Development, 80, 562-577.

Masten, A. S., Hubbard, J. J., Gest, S. D., Tellegen, A., Garmezy, N., \& Ramirez, M. (1999). Competence in the context of adversity: Pathways to resilience and maladaptation from childhood to late adolescence. Development and Psychopathology, 11, 143-169.

McClelland, M. M., Cameron, C. E., Connor, C. M., Farris, C. L., Jewkes, A. M., \& Morrison, F. J. (2007). Links between behavioral regulation and preschoolers' literacy, vocabulary, and math skills. Developmental Psychology, 43, 947.

Medin, D. L., Ross, B. H., \& Markman, A. B. (2001). Cognitive psychology. Orlando: Harcourt. 
Melhuish, E., Quinn, L., Hanna, K., Sylva, K., Sammons, P., Siraj-Blatchford, I. \& Taggart, B. (2006). Effective Pre-School Provision in Northern Ireland (EPPNI) summary report 1998-2004 (research report No. 41). Bangor: DENI.

Nisbett, R. E., Aronson, J., Blair, C., Dickens, W., Flynn, J., Halpern, D. F., \& Turkheimer, E. (2012). Intelligence: New findings and theoretical developments. American Psychologist, 67, 130-159.

Platt, L. (2007). Poverty and ethnicity in the UK. Bristol, UK: Policy Press.

Plewis, I. (2007). The Millennium Cohort Study: Technical report on sampling (4 ${ }^{\text {th }}$ ed.). London: Centre for Longitudinal Studies, Institute of Education, University of London. Reiss, F. (2013). Socioeconomic inequalities and mental health problems in children and adolescents: a systematic review. Social Science and Medicine, 90, 24-31.

Rubin, D. B. (1996). Multiple imputation after 18+ years. Journal of the American Statistical Association, 91, 473-489.

Riglin, L., Collishaw, S., Shelton, K. H., McManus, I. C., Ng-Knight, T., Sellers, R., Thapar, A. K., Frederickson, N., \& Rice, F. (2015, Early View). Higher cognitive ability buffers stress-related depressive symptoms in adolescent girls. Development and Psychopathology, 1-13.

Rutter, M. (1978). Family, area, and school influences in the genesis of conduct disorders. In L.A. Hersoy \& D. Shaffer (Eds.), Aggression and anti-social behavior in childhood and adolescence (pp. 95-114). Oxford: Pergamon Press.

Rutter, M. (2013). Annual Research Review: resilience--clinical implications. Journal of Child Psychology and Psychiatry, 54, 474-487.

Sammons, P., Sylva, K., Melhuish, E., Siraj-Blatchford, I., Taggart, B., Elliot, K., \& Marsh, A. (2004). The continuing effects of pre-school education at age 7 years (technical paper 11). London: DfES. 
Stone, L. L., Otten, R., Engels, R. C., Vermulst, A. A., \& Janssens, J. M. (2010). Psychometric properties of the parent and teacher versions of the strengths and difficulties questionnaire for 4-to 12-year-olds: A review. Clinical Child and Family Psychology Review, 13, 254-274.

Tiet, Q. Q., Bird, H. R., Davies, M., Hoven, C., Cohen, P., Jensen, P. S., \& Goodman, S. (1998). Adverse life events and resilience. Journal of the American Academy of Child and Adolescent Psychiatry, 37, 1191-1200.

von Stumm, S., \& Plomin, R. (2015). Socioeconomic status and the growth of intelligence from infancy through adolescence. Intelligence, 48, 30-36.

Weeks, M., Wild, T. C., Ploubidis, G. B., Naicker, K., Cairney, J., North, C. R., \& Colman, I. (2014). Childhood cognitive ability and its relationship with anxiety and depression in adolescence. Journal of Affective Disorders, 152, 139-145. 


\section{Acknowledgements}

This research was supported by a UK Economic and Social Research Council grant (ES/J001414/1). 
Table 1

Model Summary

\begin{tabular}{|c|l|}
\hline Model & Variables \\
\hline $\begin{array}{c}\text { (unconditional) } \\
\mathbf{2}\end{array}$ & Age (centred) in years and age-squared \\
\hline $\mathbf{3}$ & Model 1 + sampling design variables ('strata') + SED + SED*age + SED*age ${ }^{2}+$ ALE + ALE*age + ALE*age ${ }^{2}+$ NMI + NMI*age + NMI*age ${ }^{2}$ \\
\hline $\mathbf{4}$ & Model 2 + child factors ${ }^{\text {a }}+$ parent factors $^{\text {b }}$ \\
\hline $\mathbf{5}$ & Model 3 + IQ + IQ*age + IQ*age ${ }^{2}$ \\
\hline $\mathbf{6}$ & Model 4 + SED*IQ + SED*IQ*age + SED*IQ*age ${ }^{2}$ \\
\hline $\mathbf{7}$ & Model 5 + ALE*IQ + ALE*IQ*age + ALE*IQ*age ${ }^{2}$ \\
\hline
\end{tabular}

Note $:$ SED = Socio-economic disadvantage; ALE = Adverse life events; NMI = Neighbourhood median income;

${ }^{a}$ gender, ethnicity and self-regulation (time-varying);

bmother's education (whether University-educated or not by Sweep 4) and how often the parent reads to the child (time-varying). 


\section{Table 2}

Descriptives of Study Variables in the Analytic and Non-analytic Samples

\begin{tabular}{|c|c|c|c|c|c|}
\hline & \multicolumn{2}{|c|}{ Analytic sample $(\mathrm{n}=16,916)^{\mathrm{b}}$} & \multicolumn{2}{|c|}{ Non-analytic sample $(\mathrm{n}=2,328)^{\mathrm{b}}$} & \multirow{2}{*}{ Test } \\
\hline & \multicolumn{4}{|c|}{ Categorical variables } & \\
\hline & $N$ & $\%$ & $n$ & $\%$ & $F^{a}$ \\
\hline \multicolumn{6}{|l|}{ Child } \\
\hline Girl & 8,288 & 47.97 & 1,061 & 37.60 & 2.79 \\
\hline Ethnicity & & & & & $15.31 * * *$ \\
\hline White & 14,062 & 66.01 & 1,679 & 17.04 & \\
\hline Black & 596 & 8.00 & 133 & 28.68 & \\
\hline Indian & 430 & 4.53 & 67 & 7.23 & \\
\hline Pakistani/Bangladeshi & 1,077 & 10.72 & 273 & 28.87 & \\
\hline Mixed & 512 & 6.06 & 82 & .23 & \\
\hline Other & 230 & 4.67 & 73 & 17.95 & \\
\hline \multicolumn{6}{|l|}{ Parent/household } \\
\hline \multirow[t]{3}{*}{ Mother is University-educated } & 2,821 & 6.18 & 205 & 3.95 & 2.85 \\
\hline & \multicolumn{5}{|c|}{ Continuous variables } \\
\hline & $\mathbf{N}$ & $\mathbf{M}(\mathbf{S D})$ & $\mathbf{n}$ & $\mathbf{M}(\mathbf{S D})$ & $T$ \\
\hline \multicolumn{6}{|l|}{ Child } \\
\hline \multicolumn{6}{|l|}{ Internalising problems } \\
\hline Age 3 & 14577 & $2.87(2.49)$ & - & - & - \\
\hline Age 5 & 14681 & $2.50(2.52)$ & - & - & - \\
\hline Age 7 & 13415 & $2.82(2.85)$ & - & - & - \\
\hline \multicolumn{6}{|l|}{ Externalising problems } \\
\hline Age 3 & 14594 & $6.75(3.82)$ & - & - & - \\
\hline Age 5 & 14648 & $4.81(3.43)$ & - & - & - \\
\hline Age 7 & 13413 & $4.88(3.65)$ & - & - & - \\
\hline \multicolumn{6}{|l|}{ Age (years) } \\
\hline Age 3 & 15369 & $3.14(0.20)$ & 212 & $3.22(0.31)$ & $12.66^{* * *}$ \\
\hline Age 5 & 15102 & $5.21(0.24)$ & 142 & $5.17(0.31)$ & 1.54 \\
\hline Age 7 & 13765 & $7.24(0.26)$ & 92 & $7.23(0.25)$ & 0.01 \\
\hline
\end{tabular}




\begin{tabular}{|c|c|c|c|c|c|}
\hline \multicolumn{6}{|l|}{ Self-regulation } \\
\hline Age 3 & 14824 & $2.46(0.35)$ & 13 & $1.74(0.67)$ & $13.84 * * *$ \\
\hline Age 5 & 14766 & $2.52(0.35)$ & 8 & $1.85(0.15)$ & $19.81 * * *$ \\
\hline Age 7 & 13484 & $2.50(0.37)$ & 4 & $2.53(0.57)$ & 0.01 \\
\hline \multicolumn{6}{|l|}{ IQ } \\
\hline Age 3 & 13451 & $101.12(18.48)$ & 106 & $79.94(19.53)$ & $10.85^{* * *}$ \\
\hline Age 5 & 14750 & $100.98(18.90)$ & 113 & $79.58(24.58)$ & $8.15 * * *$ \\
\hline Age 7 & 13220 & $100.05(20.53)$ & 86 & $80.68(20.01)$ & $8.78 * * *$ \\
\hline \multicolumn{6}{|c|}{ Parent/household } \\
\hline \multicolumn{6}{|c|}{ Adverse life events } \\
\hline Age 3 & 16916 & $1.61(1.16)$ & 2328 & $1.12(1.30)$ & $5.05 * * *$ \\
\hline Age 5 & 16916 & $1.45(1.07)$ & 2328 & $1.48(1.10)$ & 0.21 \\
\hline Age 7 & 16916 & $1.45(1.03)$ & 2328 & $1.56(0.93)$ & 1.15 \\
\hline \multicolumn{6}{|c|}{ Socio-economic disadvantage } \\
\hline Age 3 & 12909 & $0.84(1.14)$ & 94 & $2.06(1.50)$ & $7.15 * * *$ \\
\hline Age 5 & 12806 & $0.86(1.12)$ & 60 & $2.15(1.31)$ & $7.66 * * *$ \\
\hline Age 7 & 13607 & $0.86(1.12)$ & 75 & $1.76(1.08)$ & $5.97 * * *$ \\
\hline \multicolumn{6}{|c|}{ Neighbourhood median income } \\
\hline Age 3 & 15375 & $25226.78(8804.01)$ & 214 & 19584.07(7054.35) & $6.89 * * *$ \\
\hline Age 5 & 15103 & 24645.38 (8930.79) & 142 & $19009.73(5973.75)$ & $5.88 * * *$ \\
\hline Age 7 & 13763 & $26450.63(9835.51)$ & 92 & 23620.55 (8348.77) & $2.07 *$ \\
\hline \multicolumn{6}{|c|}{ (Less) parental involvement } \\
\hline Age 3 & 15271 & $1.76(1.16)$ & 167 & $3.29(2.31)$ & $7.80 * * *$ \\
\hline Age 5 & 15053 & $1.78(1.02)$ & 119 & $3.17(2.21)$ & $6.71 * * *$ \\
\hline Age 7 & 13091 & $2.08(1.21)$ & 63 & $3.79(2.06)$ & $5.15^{* * * *}$ \\
\hline
\end{tabular}

Note: $* p<.05, * * p<.01, * * * p<.001$.

${ }^{\mathrm{a}} \mathrm{F}$ (for categorical variables) $=\mathrm{F}$ statistic for design-based Pearson chi-square that is converted to F test to account for the MCS sampling design.

${ }^{\text {b}}$ The analytic sample comprised those children who, in at least one sweep, had data for the SDQ internalising scale or for the SDQ externalising scale. The nonanalytic sample comprised children with no SDQ externalising or internalising. Proportions are weighted to account for sampling design and non-response in MCS. Ns are unweighted. 


\section{Table 3}

Correlations among the Main Variables (Risk Factors, IQ, and Externalising and Internalising Problems) in the Analytic Sample $\begin{array}{llllllllllllllllll}\text { Ext2 } & \text { Ext3 } & \text { Ext4 } & \text { Int2 } & \text { Int3 } & \text { Int4 } & \underset{2}{\text { SED }} & \underset{3}{\text { SED }} & \underset{4}{\text { SED }} & \underset{2}{\text { ALE }} & \underset{3}{\text { ALE }} & \text { ALE4 } & \text { NMI2 } & \text { NMI3 } & \text { NMI4 } & \text { IQ2 } & \text { IQ3 } & \text { IQ4 }\end{array}$

Ext2 1

Ext3

$.61 \quad 1$

Ext4

Int2

Int3

Int4

$\begin{array}{llllllll}\text { SED2 } & .27 & .26 & .24 & .23 & .23 & .22 & 1\end{array}$

$\begin{array}{lllllllll}\text { SED3 } & .25 & .26 & .24 & .22 & .22 & .23 & .84 & 1\end{array}$

$\begin{array}{llllllllll}\text { SED4 } & .25 & .26 & .24 & .22 & .23 & .23 & .77 & .83 & 1\end{array}$

$\begin{array}{lllllllllll}\text { ALE2 } & .16 & .09 & .09 & .10 & .07 & .09 & .23 & .12 & .12 & 1\end{array}$

$\begin{array}{llllllllllll}\text { ALE3 } & .10 & .14 & .12 & .06 & .12 & .13 & .10 & .21 & .16 & .13 & 1\end{array}$

$\begin{array}{llllllllllllll}\text { ALE4 } & .07 & .09 & .16 & .05 & .07 & .16 & .05 & .08 & .20 & .13 & .30 & 1\end{array}$

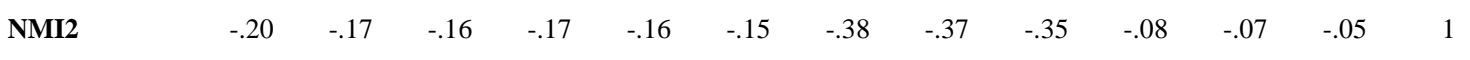

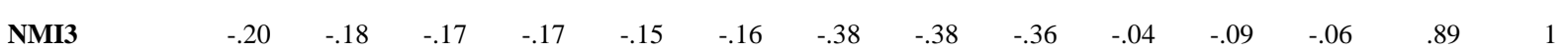

NMI4

IQ2

IQ3

$\begin{array}{llllllllllllllll}-.15 & -.14 & -.13 & -.12 & -.12 & -.11 & -.32 & -.32 & -.30 & -.03 & -.06 & -.09 & .79 & & .82\end{array}$

$\begin{array}{llllllllllllll}-.28 & -.26 & -.24 & -.23 & -.21 & -.20 & -.36 & -.35 & -.34 & -.05 & -.03 & -.02 & .27\end{array}$

$\begin{array}{llllllllllll}-.23 & -.25 & -.24 & -.17 & -.19 & -.19 & -.29 & -.29 & -.28 & -.04 & -.10 & -.02\end{array}$


Notes: All significant at $p<.05 .2,3$, and 4 refer to MCS sweeps. SED = Socio-economic disadvantage; ALE = Adverse life events; NMI = Neighbourhood median income; Ext = Externalising; Int = Internalising; IQ=Intelligence. 


\section{Table 4}

Fixed Effects Estimates and Variance Covariance Estimates of Trajectories of Internalising and Externalising Problems in the Analytic Sample (Age Centred at 5.22 Years)

\begin{tabular}{|c|c|c|c|c|c|c|c|c|c|c|c|c|}
\hline & \multicolumn{6}{|c|}{ Model 1 (unconditional) } & \multicolumn{6}{|c|}{ Model 4} \\
\hline & \multicolumn{3}{|c|}{ Internalising } & \multicolumn{3}{|c|}{ Externalising } & \multicolumn{3}{|c|}{ Internalising } & \multicolumn{3}{|c|}{ Externalising } \\
\hline & Coeff. & SE & $95 \% \mathrm{CI}$ & Coeff. & SE & $95 \% \mathrm{CI}$ & Coeff. & SE & $95 \% \mathrm{CI}$ & Coeff. & SE & $95 \% \mathrm{CI}$ \\
\hline \multicolumn{13}{|c|}{ Fixed effects } \\
\hline Constant & $2.504 * * *$ & 0.033 & {$[-2.44,-2.57]$} & $4.840 * * *$ & 0.046 & {$[4.75,4.93]$} & $8.830 * * *$ & 0.615 & {$[7.63,10.04]$} & $16.338 * * *$ & 0.770 & {$[14.83,17.85]$} \\
\hline Age & $-0.040 * * *$ & 0.006 & {$[-0.05,-0.03]$} & $-0.477 * * *$ & 0.008 & {$[-0.49,-0.46]$} & $-0.591 * *$ & 0.185 & {$[-0.95,-0.23]$} & $-1.447 * * *$ & 0.230 & {$[-1.90,-1.00]$} \\
\hline $\mathrm{Age}^{2}$ & $0.064 * * *$ & 0.004 & {$[0.06,0.07]$} & $0.203 * * *$ & 0.005 & {$[0.19,0.21]$} & 0.200 & 0.130 & {$[-0.05,0.45]$} & $0.639 * * *$ & 0.151 & {$[0.35,0.94]$} \\
\hline Girl & & & & & & & -0.026 & 0.030 & {$[-0.09,0.04]$} & $-0.768 * * *$ & 0.042 & {$[-0.85,-0.69]$} \\
\hline \multicolumn{13}{|l|}{ Ethnicity (Ref: White) } \\
\hline Mixed & & & & & & & 0.130 & 0.094 & {$[-0.05,0.31]$} & 0.087 & 0.129 & {$[-0.17,0.34]$} \\
\hline Indian & & & & & & & $0.521 * * *$ & 0.113 & {$[0.30,0.74]$} & -0.112 & 0.153 & {$[-0.41,0.19]$} \\
\hline Pakistani/Bangladeshi & & & & & & & $1.000 * * *$ & 0.087 & {$[0.83,1.17]$} & $-0.308 *$ & 0.120 & {$[-0.54,-0.07]$} \\
\hline Black & & & & & & & -0.079 & 0.098 & {$[-0.27,0.11]$} & $-0.945^{* * *}$ & 0.134 & {$[-1.21,-0.68]$} \\
\hline Other & & & & & & & $0.881 * * *$ & 0.145 & {$[0.60,1.17]$} & -0.309 & 0.199 & {$[-0.70,0.08]$} \\
\hline Self-regulation & & & & & & & $-0.242 * * *$ & 0.007 & {$[-0.26,-0.23]$} & $-0.454 * * *$ & 0.009 & {$[-0.47,-0.44]$} \\
\hline Mother is University-educated & & & & & & & $-0.209 * * *$ & 0.047 & {$[-0.30,-0.12]$} & $-0.768 * * *$ & 0.060 & {$[-0.89,-0.65]$} \\
\hline (Low) parental involvement & & & & & & & $0.047 * * *$ & 0.010 & {$[0.03,0.07]$} & $0.140 * * *$ & 0.013 & {$[0.114,0.165]$} \\
\hline SED & & & & & & & $0.238 * * *$ & 0.018 & {$[0.20,0.27]$} & $0.359 * * *$ & 0.023 & {$[0.315,0.403]$} \\
\hline SED*age & & & & & & & $0.015^{*}$ & 0.006 & {$[0.003,0.03]$} & -0.011 & 0.008 & {$[-0.03,0.004]$} \\
\hline
\end{tabular}


SED*age $^{2}$

ALE

ALE*age

ALE*age $^{2}$

NMI

NMI*age

NMI* age ${ }^{2}$

IQ

IQ*age

$\mathrm{IQ}^{*} \mathrm{age}^{2}$

Area stratum (Ref: England-

advantaged)

England-disadvantaged

England-ethnic

Scotland-advantaged

Scotland-disadvantaged

Northern Ireland-advantaged

Northern Ireland-disadvantaged

Wales-advantaged

Wales-disadvantaged

\begin{tabular}{|c|c|c|c|c|c|}
\hline 0.002 & 0.004 & {$[-0.01,0.01]$} & -0.004 & 0.005 & {$[-0.01,0.01]$} \\
\hline $0.113 * * *$ & 0.016 & {$[0.08,0.14]$} & $0.128 * * *$ & 0.019 & {$[0.09,0.16]$} \\
\hline $0.034 * * *$ & 0.006 & {$[0.003,0.03]$} & $-0.018 *$ & 0.008 & {$[-0.03,-0.004]$} \\
\hline $0.009 *$ & 0.004 & {$[0.001,0.02]$} & $0.025 * * *$ & 0.005 & {$[0.02,0.04]$} \\
\hline$-0.287 * * *$ & 0.060 & {$[-0.40,0.17]$} & $-0.432 * * *$ & 0.075 & {$[-0.58,-0.29]$} \\
\hline $0.054 * *$ & 0.019 & {$[0.02,0.09]$} & $0.124 * * *$ & 0.024 & {$[0.08,0.17]$} \\
\hline-0.006 & 0.013 & {$[-0.03,0.02]$} & $-0.036^{*}$ & 0.015 & {$[-0.07,-0.01]$} \\
\hline$-0.010 * * *$ & 0.001 & {$[-0.01,-0.007]$} & $-0.019 * * *$ & 0.001 & {$[-0.022,-0.016]$} \\
\hline-0.0002 & 0.001 & {$[-0.001,0.001]$} & -0.0003 & 0.001 & {$[-0.001,-0.001]$} \\
\hline$-0.001 * *$ & 0.0003 & {$[-0.002,-0.0003]$} & $-0.001 * * *$ & 0.0004 & {$[-0.002,-0.001]$} \\
\hline $0.269 * * *$ & 0.051 & {$[0.17,0.37]$} & $0.332 * * *$ & 0.070 & {$[0.20,0.47]$} \\
\hline $0.252 * *$ & 0.082 & {$[0.09,0.41]$} & 0.107 & 0.113 & {$[-0.11,0.33]$} \\
\hline-0.112 & 0.076 & {$[-0.26,0.04]$} & $-0.250 *$ & 0.104 & {$[-0.45,-0.05]$} \\
\hline-0.047 & 0.079 & {$[-0.20,0.11]$} & 0.031 & 0.108 & {$[-0.18,0.24]$} \\
\hline-0.092 & 0.092 & {$[-0.27,0.09]$} & $-0.437 * * *$ & 0.126 & {$[-0.68,0.19]$} \\
\hline 0.053 & 0.079 & {$[-0.11,0.21]$} & -0.174 & 0.108 & {$[-0.39,0.04]$} \\
\hline$-0.172 *$ & 0.087 & {$[-0.34,0.003]$} & -0.158 & 0.118 & {$[-0.39,0.07]$} \\
\hline-0.052 & 0.066 & {$[-0.08,0.18]$} & $0.186^{*}$ & 0.090 & {$[0.01,0.36]$} \\
\hline
\end{tabular}

\section{Random effects}


Level 3 (ward)

Intercept

Level 2 (child)

Intercept

Slope

Intercept/slope covariance

Slope
Level 1 (occasion)

$0.252 * * *$

$0.026[0.21,0.31]$

$0.489 * * * \quad 0.056$

$[0.39,0.61]$

$0.018 * * *$

0.007

$[0.01,0.04]$

$0.036^{* * *}$

0.015

[0.02,0.08]

\begin{tabular}{|c|c|c|c|c|c|c|c|c|c|c|c|}
\hline $3.469 * * *$ & 0.054 & {$[3.36,3.58]$} & $8.116^{* * * *}$ & 0.111 & {$[7.90,8.34]$} & $2.793 * * *$ & 0.047 & {$[2.70,2.89]$} & $5.650 * * *$ & 0.086 & {$[5.49,5.82]$} \\
\hline $.161 * * *$ & 0.008 & {$[0.15,0.18]$} & $0.313 * * *$ & 0.012 & {$[0.29,0.34]$} & $0.143 * * *$ & 0.008 & {$[0.13,0.16]$} & $0.279 * * *$ & 0.011 & {$[0.26,0.30]$} \\
\hline O & 0.013 & & $-0.064 * *$ & 0.023 & & $0.163^{* *}$ & 0.012 & & $-0.187 * *$ & 0.020 & \\
\hline
\end{tabular}

Note: $* p<.05, * * p<.01, * * * p<.001$. SED = Socio-economic disadvantage ALE = Adverse life events; NMI = Neighbourhood median income; Ns $=16,877$ (externalising), 16,884 (internalising). $\mathrm{N}$ is not 16,916 because we did not impute missing values on the outcomes (externalising and internalising). 


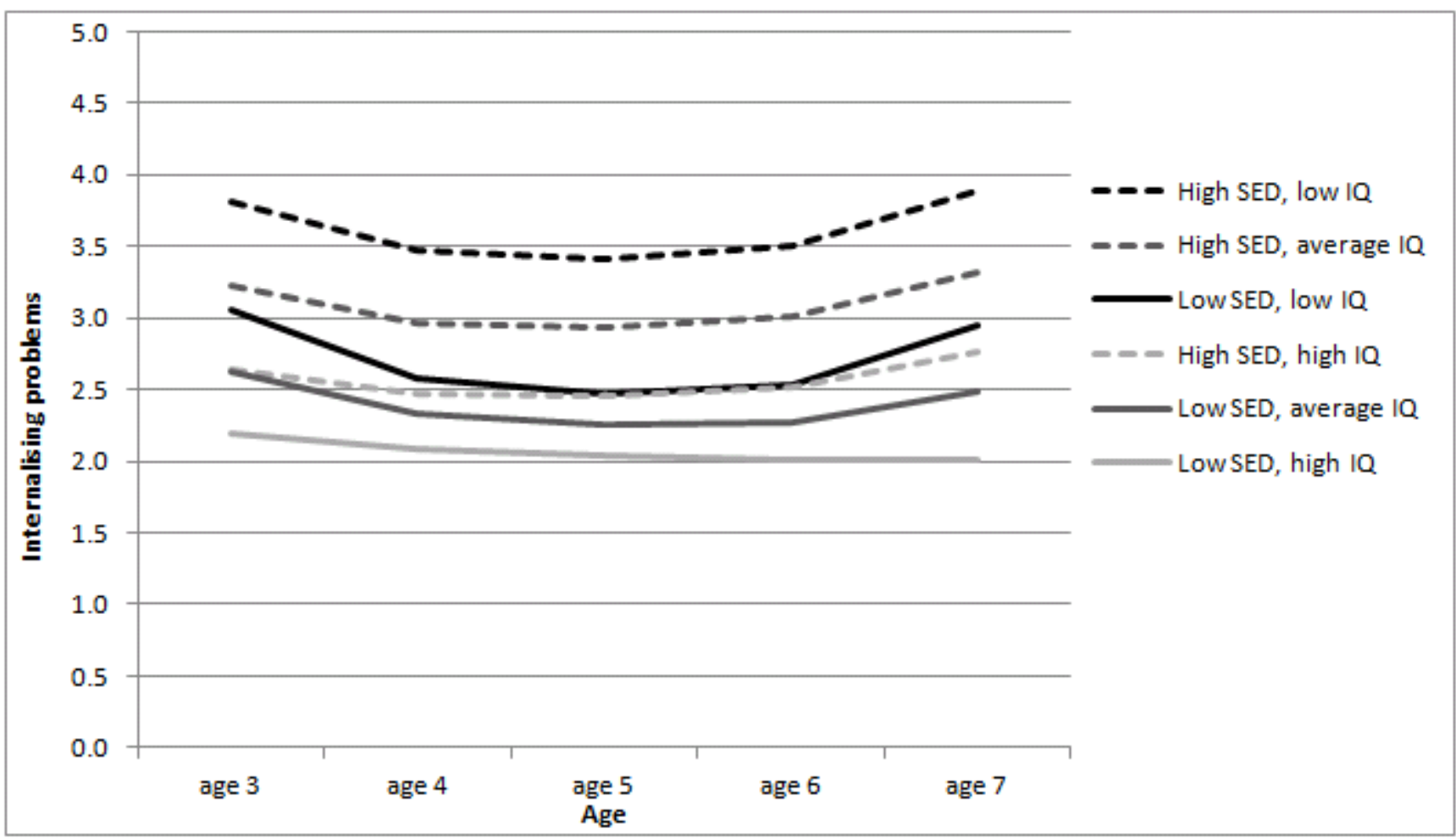

Figure 1. Predicted internalising score trajectories by high and low family socio-economic disadvantage (SED) and low, average and high IQ 


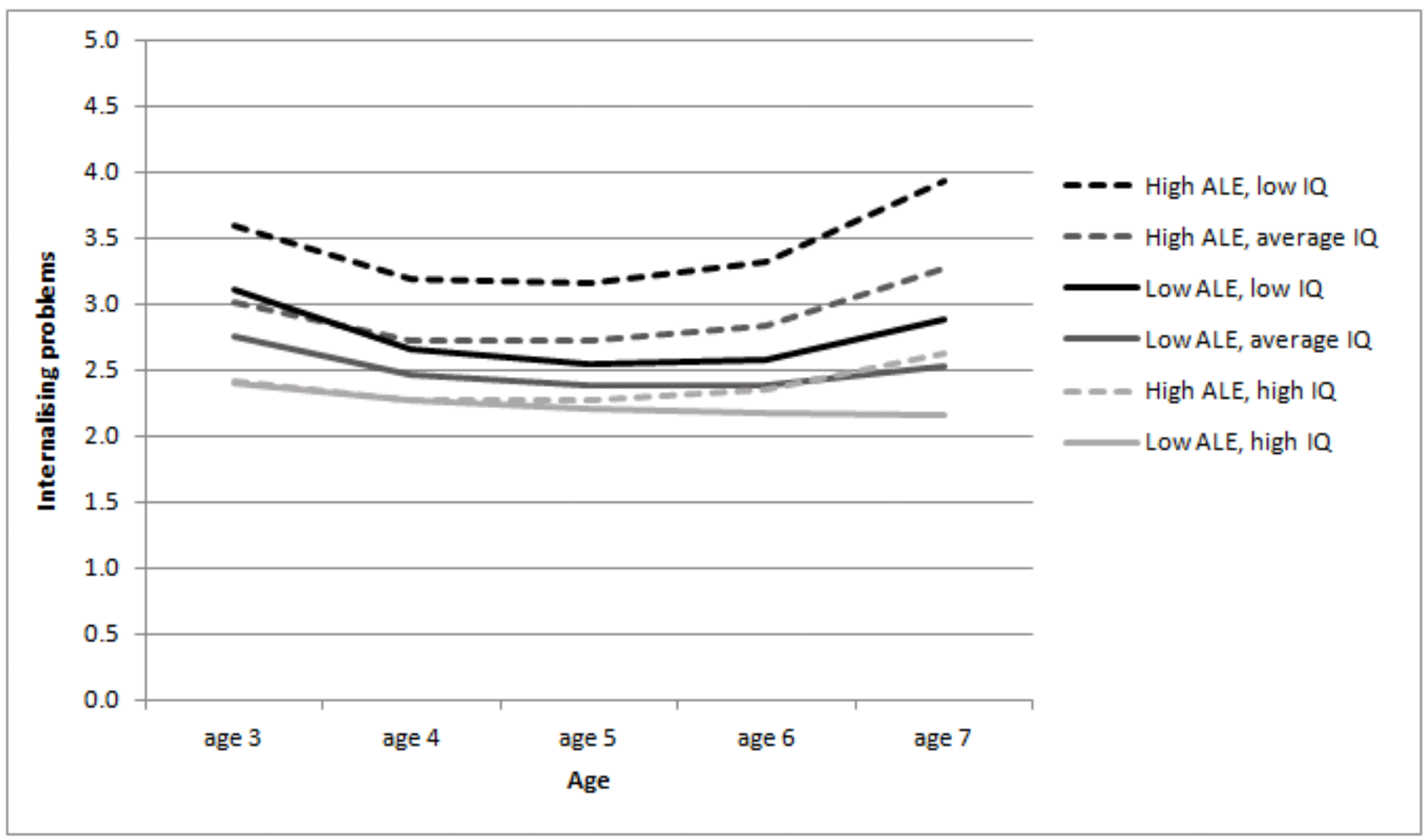

Figure 2. Predicted internalising score trajectories by high and low number of adverse life events (ALE), and low, average and high IQ 


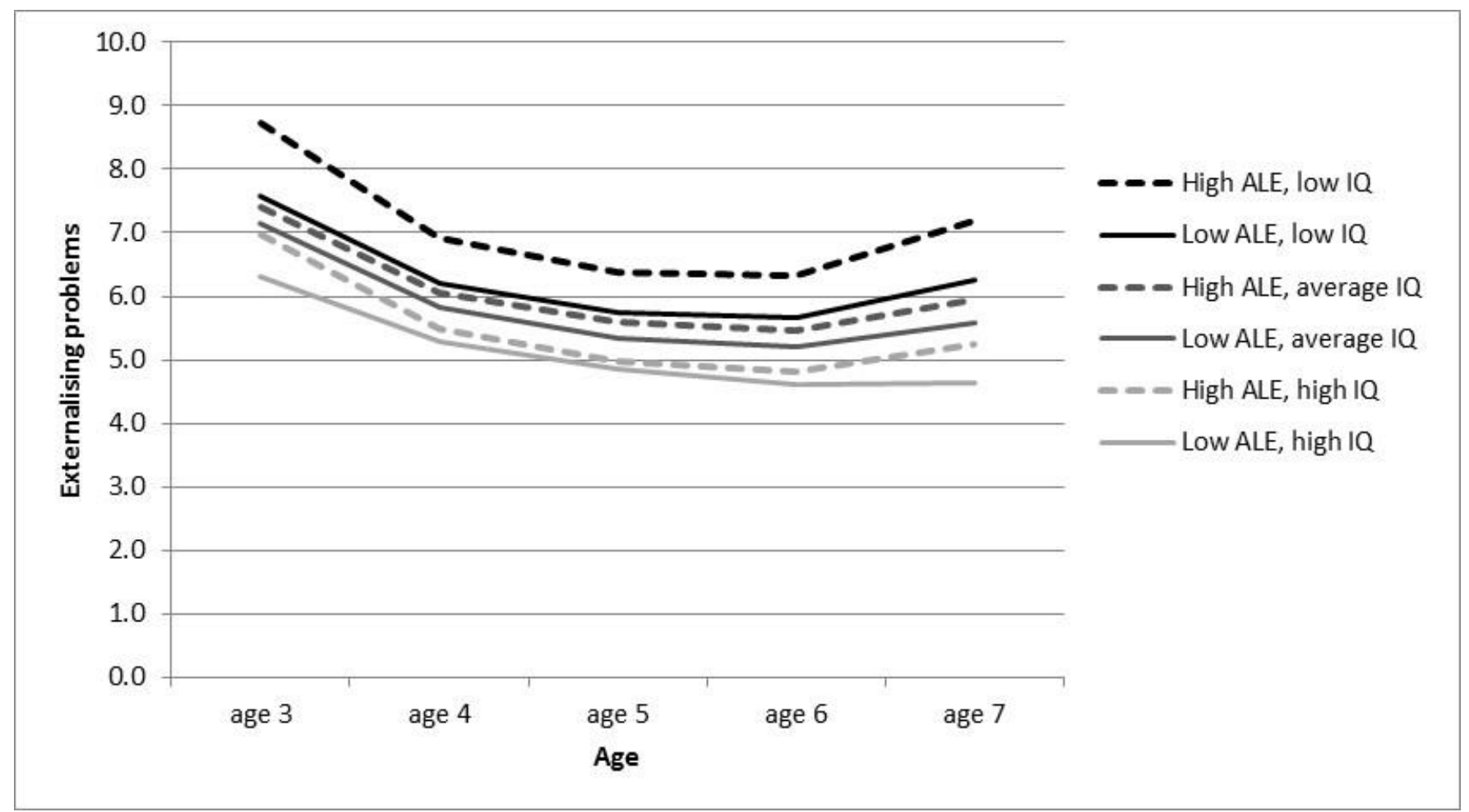

Figure 3. Predicted externalising score trajectories by high and low number of adverse life events (ALE), and low, average and high IQ 


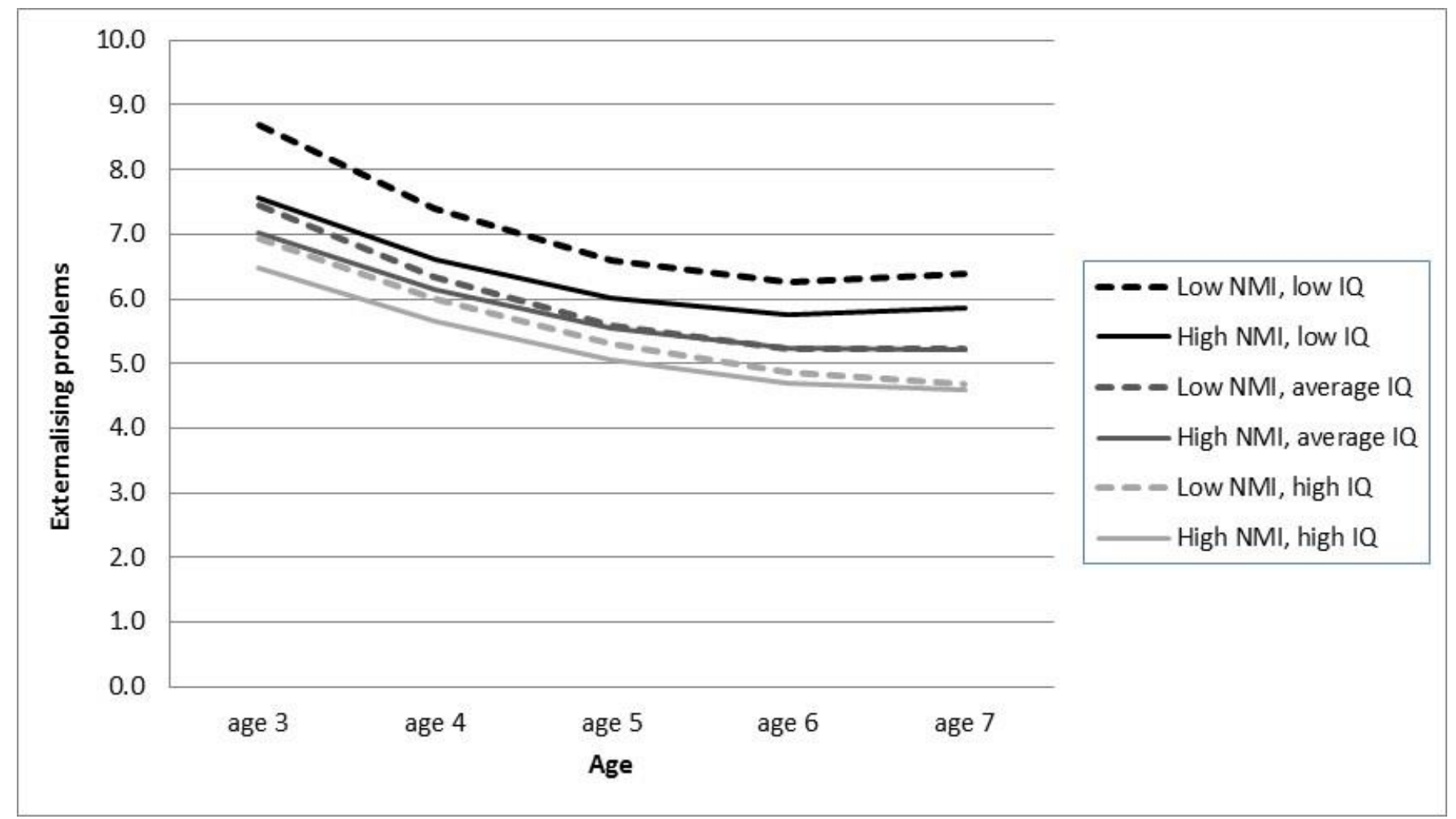

Figure 4. Predicted externalising score trajectories by high and low neighbourhood median income (NMI), and low, average and high IQ 